\title{
Immunohistochemical expression of chemokine receptor in neuroendocrine neoplasms (CXCR4) of the gastrointestinal tract: a retrospective study of 71 cases
}

\author{
OANa Popa ${ }^{1,2)}$, Sorina Maria TĂban ${ }^{1,2)}$, Alis Liliana CARMen Dema ${ }^{1,2)}$, ANDRei Dorel Plopeanu ${ }^{3)}$ \\ ROBERT ALEXANDRu BARNA ${ }^{2,4)}$, MĂRIOARA CORNIANU ${ }^{1,2)}$, SORIN DEMA ${ }^{5)}$ \\ 1) Discipline of Morphopathology, Department of Microscopic Morphology, Victor Babeş University of Medicine and Pharmacy, \\ Timişoara, Romania \\ 2) Anapatmol Research Center, Victor Babeş University of Medicine and Pharmacy, Timişoara, Romania \\ 3) Department of Pathology, Pius Brînzeu Emergency County Hospital, Timişoara, Romania \\ 4) Discipline of Gastroenterology and Hepatology, Department of Internal Medicine II, Victor Babeş University of Medicine and \\ Pharmacy, Timişoara, Romania \\ ${ }^{5)}$ Service of Radiotherapy, Emergency City Hospital, Timişoara, Romania
}

\begin{abstract}
Aim: C-X-C motif chemokine receptor 4 (CXCR4) is expressed in many tumor entities, including gastrointestinal neuroendocrine neoplasms (GI-NENs). However, the role of CXCR4 expression in GI-NENs has been less studied. Our objective was to investigate the expression of CXCR4 in a series of GI-NENs with various clinical and pathological features. Methods: The immunohistochemical (IHC) expression of CXCR4 (clone UMB2) was examined in 71 GI-NENs and a semiquantitative immunoreactivity score (IRS) was calculated taking into consideration the intensity of the IHC reaction and the percentage of the tumor cells which showed positive expression. Results were compared with several clinical and pathological prognostic factors. Results: High CXCR4 expression was noted in 31 (43.7\%) cases. Low IRS values were more frequent in NENs from the small intestine $(66.7 \%)$ and stomach $(60 \%)$. Also, all appendix tumors had IRS value of zero. High CXCR4 expression was noticed in $52.5 \%$ of liver metastases, compared to $40.4 \%$ primary tumors. A significant relationship was observed between the CXCR4 expression and the tumor grade $(p=0.0216)$, and high IRS value was correlated with clinical stages III and IV $(p=0.0142)$ and lympho-vascular invasion ( $p=0.0129$ ). $74.1 \%$ of $\mathrm{G} 1$ neuroendocrine tumors (NETs) had a low IRS, G3 NETs showed minor differences between low (42.9\%) and high (57.1\%) expression and $66.7 \%$ of neuroendocrine carcinomas (NECs) presented high expression of CXCR4. Conclusions: The present study highlighted that high CXCR4 expression is associated with high grade and advanced stage GI-NENs, as well as with metastatic cases. In these cases, high CXCR4 expression could serve as an important target for CXCR4 antagonists.
\end{abstract}

Keywords: CXCR4, chemokine receptor, neuroendocrine tumor, neuroendocrine carcinoma, gastrointestinal tract.

\section{a Introduction}

Neuroendocrine neoplasms (NENs) are a group of tumors which develop from the neuroendocrine cell system, can involve almost all anatomical sites and present various clinical manifestations associated with the production of specific hormones [1-3]. Gastro-entero-pancreatic NENs (GEP-NENs) account for 70\% of NENs, most commonly reported in the small bowel (38\%) and rectum (34.4\%), and less frequently in the esophagus or anus [1,4].

In 2019, the World Health Organization (WHO) adopted a new classification for the gastrointestinal NENs (GINENs), which is similar, along with the tumor grading, with the one discussed in the 2017 WHO Classification of pancreatic NENs, defining a new category - the welldifferentiated grade 3 neuroendocrine tumors (NETs) of the digestive system. Therefore, in the current $W H O$ Classification, NETs have three grades of differentiation (G1, G2 and G3), while neuroendocrine carcinomas (NECs) are by definition high-grade tumors, continuing to be grouped into small-cell NECs (SCNECs) and large-cell
NECs (LCNECs) [5]. Genetic studies of GI-NENs reported molecular differences between NETs, which usually harbor menin 1 (MENI), death domain-associated protein $(D A X X)$ and alpha-thalassemia/mental retardation, X-linked (ATRX) mutations and NECs, which are characterized by tumor protein p53 (TP53) and retinoblastoma $1(R B 1)$ mutations [6]. Also, a difference between G3 NETs and NECs resides from the successful response of NECs to platinum-containing therapy, while G3 NETs patients do not respond to this chemotherapy, but have longer survival and better outcome [7].

Another update consists of the new defined mixed neuroendocrine non-NENs (MiNENs), former called mixed adeno-NECs (MANECs), in which neuroendocrine and nonneuroendocrine components represents each more than $30 \%$ of the tumor cells [2].

GEP-NENs are usually confirmed by immunohistochemistry using chromogranin $\mathrm{A}(\mathrm{CgA})$ and synaptophysin (Syn). Furthermore, immunohistochemical (IHC) markers could be used in NENs to determine the origin of some tumors [cytokeratin (CK) 7, CK20, caudal type homeobox 2

This is an open-access article distributed under the terms of a Creative Commons Attribution-NonCommercial-ShareAlike 4.0 International Public License, which permits unrestricted use, adaptation, distribution and reproduction in any medium, non-commercially, provided the new creations are licensed under identical terms as the original work and the original work is properly cited. 
(CDX2), thyroid transcription factor-1 (TTF-1)], to differentiate NEC from G3 NETs (p53, Rb1) or to identify patients who could benefit from targeted therapy, e.g., expression of somatostatin receptors (SSTRs) $[1,7]$. Apart from the use of SSTRs, mainly expressed by G1 and G2 NETs, there is a promising role for a novel marker, the C-X-C motif chemokine receptor 4 (CXCR4), which is associated more frequently with NEC, but can also be positive in NETs. Elevated expression of CXCR4 is associated with high risk for early metastasis, tumor progression, tumor recurrence and poor patient outcome [8]. From the clinical point of view, CXCR4-positive NENs could benefit from treatment with CXCR4 antagonists which display a high anti-proliferative capacity in animal tumor models [7].

Adenocarcinoma (ADK) ex-goblet cell carcinoids (AGCCs) or mixed goblet cell carcinoid-ADK is an enigmatic entity, an amphicrine tumor with glandular or mucinous and neuroendocrine differentiation, which shows focal positive expression of $\mathrm{CgA}$ and other neuroendocrine markers. In the current $W H O$ Classification, these neoplasms are classified as goblet cell ADKs [9].

Currently, there are a limited number of studies which assess the IHC expression of CXCR4 in GI-NENs, although IHC allows precise evaluation of this immunomarker.

\section{Aim}

The aim of this study was to evaluate, based on IHC assay, the CXCR4 expression in GI-NENs, MiNENs and AGCCs and to correlate this expression with clinical and morphological factors that impact the overall prognosis, outcome, and treatment of the patients.

\section{Patients, Materials and Methods}

This study was performed in line with the principles of the Declaration of Helsinki. Ethics approval was obtained from the Ethics Committee of Victor Babeş University of Medicine and Pharmacy, Timişoara, Romania.

\section{Study group}

Sixty-seven patients with GI-NENs and four patients with AGCCs were recruited in the study, all of them histologically confirmed between January 2008 and December 2018 at the Department of Pathology, Pius Brînzeu Emergency County Hospital, Timişoara. All 71 cases were reclassified in accordance with the 2019 WHO Classification, as follows: 52 well-differentiated NETs, 12 SCNECs or LCNECs, three MiNENs and four cases of AGCC. Mitotic rate $>20 / 2 \mathrm{~mm}^{2}$ or Ki-67 proliferation index (Ki-67 PI) $>20 \%$ defined the well-differentiated G3 NETs and NECs. Apart from the morphology, positive expression for p53 differentiated G3 NETs from NECs $[10,11]$. Their clinical and pathological characteristics are summarized in Table 1.

\section{Histological and IHC interpretation}

IHC stainings were performed on 3-4 $\mu$ m thickness sections of formalin-fixed paraffin-embedded samples from all 71 cases, using Leica BOND-MAX and Bond Polymer Refine Detection Kit (Leica Biosystems Newcastle, UK). Leica ready-to-use (RTU) kits were used for Ki-67 (clone MM1), CgA (clone 5H7), Syn (clone 27G12) and p53 (clone DO-7), following the manufacturer protocols.
Table 1 - Main clinical and pathological features of the cases

\begin{tabular}{|c|c|}
\hline Clinical and pathological features & No. of cases $(\%)(n=71)$ \\
\hline \multicolumn{2}{|l|}{ Gender } \\
\hline Male & $37(52.1)$ \\
\hline Female & $34(47.9)$ \\
\hline \multicolumn{2}{|l|}{ Age at diagnosis [years] } \\
\hline$<20$ & $2(2.8)$ \\
\hline $20-29$ & $1(1.4)$ \\
\hline $30-39$ & $3(4.2)$ \\
\hline $40-49$ & $7(9.9)$ \\
\hline $50-59$ & $17(23.95)$ \\
\hline $60-69$ & $24(33.8)$ \\
\hline$\geq 70$ & $17(23.95)$ \\
\hline \multicolumn{2}{|l|}{ Tumor location } \\
\hline Stomach & $10(14.1)$ \\
\hline Duodenum & $2(2.8)$ \\
\hline Small intestine & $10(14.1)$ \\
\hline Appendix & $6(8.5)$ \\
\hline Right colon & $11(15.5)$ \\
\hline Left colon (including rectum) & $13(18.3)$ \\
\hline Hepatic metastasis & $19(26.7)$ \\
\hline \multicolumn{2}{|l|}{ Tumor grade } \\
\hline G1 & $28(39.4)$ \\
\hline G2 & $21(29.6)$ \\
\hline G3 & $22(31)$ \\
\hline \multicolumn{2}{|l|}{ Tumor type } \\
\hline$N E T$ & $52(73.2)$ \\
\hline G1 NET & $27(38)$ \\
\hline G2 NET & $18(25.4)$ \\
\hline G3 NET & $7(9.8)$ \\
\hline NEC & $12(17)$ \\
\hline SCNEC & $3(4.25)$ \\
\hline LCNEC & $9(12.7)$ \\
\hline MiNEN & $3(4.2)$ \\
\hline AGCC & $4(5.6)$ \\
\hline AGCC G1 & $1(1.4)$ \\
\hline AGCC G2 & $1(1.4)$ \\
\hline AGCC G3 & $2(2.8)$ \\
\hline
\end{tabular}

AGCC: Adenocarcinoma ex-goblet cell carcinoid; G: Grade; LCNEC: Large-cell neuroendocrine carcinoma; MiNEN: Mixed neuroendocrine non-neuroendocrine neoplasm; NEC: Neuroendocrine carcinoma; NET: Neuroendocrine tumor; SCNEC: Small-cell neuroendocrine carcinoma.

For the IHC detection of anti-CXCR4 antibody (clone UMB2, Abcam), the following protocol was utilized: deparaffinization of tissue sections and pretreatment with the Epitope Retrieval Solution $2(\mathrm{pH} 9)$ at $98^{\circ} \mathrm{C}$ for 20 minutes, then specimens were incubated with the primary antibody for 30 minutes, at a dilution of 1:400. Visualization was performed at room temperature for 20 minutes, utilizing the Leica Bond Polymer Refine Detection Kit. Finally, after washing in water, the sections were counterstained with Hematoxylin. High-grade urothelial carcinoma samples were used as control of the reaction. Tumor cells expressing moderate or intense cytoplasmic and/or membrane pattern, as well as positive expression of endothelial cells and lymphocytes were considered positive control.

Ki-67 PI represents the percentage of cells with nuclear expression from a total of 500 tumoral cells in hot-spots. All tumor cells were counted in biopsies where only a small number of tumor cells were present. The IHC expression 
of p53 was evaluated in all tumors with Ki-67 PI greater than $20 \%$. The IHC expression was considered positive only when the percentage of tumors cells with intense nuclear staining was above $25 \%$.

To evaluate the CXCR4 immunoexpression, we used the semiquantitative immunoreactivity score (IRS) proposed by Remmele \& Stegner, which considers the intensity of the reaction and the proportion of positive tumor cells [7, 12]. The staining intensity was assessed and grouped in four categories: no staining -0 , mild staining -1 , moderate staining -2 and strong staining -3 . The percentage of positive tumor cells was appraised in five gradations: no positive cells $-0,<10 \%$ positive cells $-1,10-50 \%$ positive cells $-2,51-80 \%$ positive cells $-3,>80 \%$ positive cells -4 . The IRS value was obtained after multiplying the staining intensity score by the positive tumor cells gradation resulting in values ranging from 0 to 12 . Cases with IRS values of 0 to 3 were considered to have a low expression, and those with IRS value between 4 to 12 were considered to have a high expression of the CXCR4 chemokine receptor.

\section{Statistical analysis}

Statistical analysis was performed using GraphPad Prism version 9.0 (GraphPad Software, Inc., San Diego, CA, USA). Descriptive statistics including patient's general data, tumor site, grade, and type, were expressed as numbers and percentages. CXCR4 immunoexpression analysis was correlated with various clinical-pathological data, such as tumor type, location, grade, and clinical stage. $\chi^{2}$ (chisquared) test was applied for the statistical evaluation and significant differences were considered when the value of $p$ was less than $0.05 \%$.

\section{ㅁ Results}

Tumor samples from 71 patients, 37 men and 34 women, were evaluated in the present investigation, with a mean age at diagnosis of 59.9 years; three patients with NETs were under the age of 30 years.

Out of the total number of cases, 52 were primary tumors of the GI tract, more frequently diagnosed in the rectum $(18.3 \%)$, right colon $(14.1 \%)$ or in the small intestine (14.1\%), and 19 were liver metastases diagnosed in core biopsies samples or in hepatic resection specimens.

Two of the three cases of MiNEN were diagnosed in the left colon (G1 ADK in association with LCNEC and G2 ADK with G2 NET) and one had a gastric localization (G2 ADK in association with G2 NET). Our study also includes four cases of AGCCs, which were reclassified as follows: two cases of G1, respectively G2 appendicular AGCC and two cases of G3 AGCC in the right colon.

The immunoreaction for CXCR4 was difficult to interpret due to the high grade of heterogeneity of the staining pattern. Most cases expressed cytoplasmic, membranous or cytoplasmic and membranous positivity and, in a few cases, there was an evident staining of the nuclei which we considered negative. Low CXCR4 immunoexpression was defined by IRS $0-3$ and high CXCR4 immunoexpression by IRS 4-12 (Figure 1).

Out of the 71 GI-NENs studied, 40 cases have low CXCR4 expression (56.3\%), and 31 cases presents high CXCR4 expression (43.7\%), as shown in Table 2. Among the patients with high expression of CXCR $4,54.8 \%$ were women and $45.2 \%$ were men, with a mean age of 61.2 years, while in the category of tumors with low CXCR4 expression, $57.5 \%$ were male patients and $42.5 \%$ were female, with a mean age of 58.9 years.

No interrelationship was observed between CXCR4 expression and tumor localization within the segments of the GI tract, although lower IRS values were noticed more frequently in NENs from the small intestine $(66.7 \%)$, stomach $(60 \%)$ and appendix (100\%). The IRS value was zero for all appendix tumors. High CXCR4 expression was noticed in $52.5 \%$ of liver metastases, more frequently than in primary tumors $(40.4 \%)$.

The $c h i$-squared test showed a significant relationship between CXCR4 expression and tumor grade $(p=0.0216)$ (Figure 2), CXCR4 was more expressed in G3 (63.3\% high IRS) than in G1 tumors (75\% low IRS). Moreover, based on tumor stage, we grouped cases in stage I and II tumors and stage III and IV tumors, in order to analyze the relationship between the tumor stage and CXCR4 expression. A significantly low IRS value was noticed for stage I-II tumors ( $p=0.0142$ ) (Figure 3 ). High expression of CXCR4 was also correlated with the presence of lymphovascular invasion $(p=0.0129)$.

An association between tumor type and CXCR4 expression is observed, even if it is not a significant one. G1 NETs presented a low expression of CXCR4 in $74.1 \%$ of cases, G3 NETs showed minor differences between low (42.9\%) and high (57.1\%) expression of CXCR4, and NECs presented a high expression in $66.7 \%$ of cases. Seven cases of G1 NETs and eight cases of G2 NETs with high CXCR4 expression were identified, despite the low Ki-67 PI, out of which 10 cases had an IRS value of 4-6 and five cases presented an IRS value of 9-12.

No relevant differences were seen between the CXCR4 expression and other parameters investigated, in particular positive regional lymph nodes or perineural invasion.

\section{ㅁ Discussions}

Chemokines, a family of cytokines secreted by epithelial and stromal cells $[13,14]$, mediate numerous cellular processes involved in cell signaling and migration. They perform their functions by interacting with specific membrane receptors, coupled with the $\mathrm{G}$ protein. Chemokines are split into four groups (CXC, CX3C, CC and C) [15] and comprise over 50 different entities discovered so far [16].

$\mathrm{C}-\mathrm{X}-\mathrm{C}$ motif chemokine ligand 12 (CXCL12) or "stromal cell-derived factor-1" binds to the CXCR4 molecule receptor or "Fusin" and activates the G proteincoupled receptor. This process triggers the increase of intracellular calcium, as well as the activation of mitogenactivated protein (MAP) kinase. All these processes contribute to the activation of the phosphoinositide 3kinase (PI3K)/Akt/mammalian target of rapamycin (mTOR) signaling pathway through a series of complex biochemical processes that stimulate cell adhesion and chemotaxis, resulting in survival, proliferation, cell adhesion and gene transcription. Although initially studied in human immunodeficiency virus (HIV) infection, CXCR4 chemokine has been researched in over 23 cancers, including chronic leukemia, breast, kidney, lung, brain, prostate, pancreatic, ovarian and melanoma, being involved in cell proliferation, tumor angiogenesis, metastasis, and the acquisition of therapeutic resistance [15].

Molecular imaging techniques have lately developed into essential clinical tools in the evaluation and quanti- 
fication of biomarkers for early assessment of therapy response [17]. This approach holds great promise for reducing the cost of specialized drug development, providing non-invasive diagnosis, directing therapy, and improving patient outcomes [18]. Recent studies analyzed the expression of CXCR4 in NETs using molecular imaging techniques. Weich et al. analyzed the potential of identifying
CXCR4-positive NETs providing two different positron emission tomography-computed tomography (PET-CT)

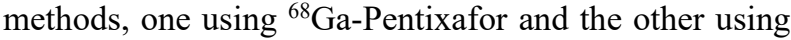
2-deoxy-2-[fluorine-18]fluoro-D-glucose $\left({ }^{18} \mathrm{~F}-\mathrm{FDG}\right)$. The study concluded that the conventional method using ${ }^{18} \mathrm{~F}$ FDG identified significant more lesions should be used for imaging CXCR4 assessment [19].

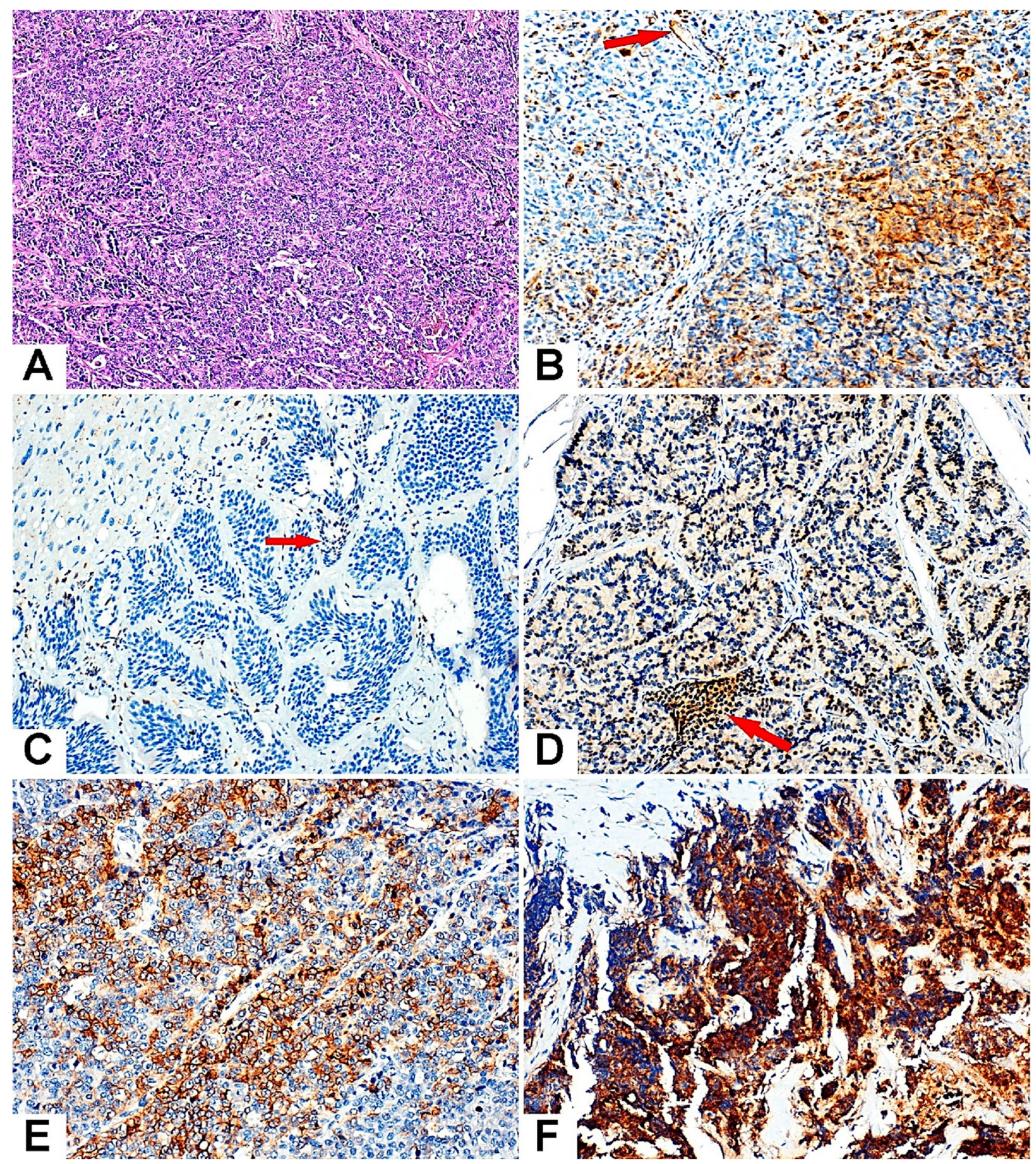

Figure 1 -Microscopic features of the NENs examined in the study. (A) LCNEC. (B) High-grade urothelial carcinoma served as positive control. Cytoplasmic and membranous positive expression was observed within tumor cells, as well as positive endothelial cells (red arrow). (C) Liver metastasis from a well-differentiated G2 NET with IRS $=0$ (the nuclear staining of rare tumor cells is not counted). (D) Well-differentiated G1 NET, IRS=8. A small group of positive intratumoral lymphocytes is marked with red arrow. (E) LCNEC, IRS=9. (F) SCNEC with intensely positive cytoplasm of the tumor cells, IRS $=12$. HE staining: $(A) \times 100$. Anti-CXCR4 chemokine receptor antibody immunomarking: $(B-F)$ ×400. CXCR4: C-X-C motif chemokine receptor 4; HE: Hematoxylin-Eosin; IRS: Immunoreactivity score; LCNEC: Large-cell neuroendocrine carcinoma; NEC: Neuroendocrine carcinoma; NENs: Neuroendocrine neoplasms; NET: Neuroendocrine tumor; SCNEC: Small-cell neuroendocrine carcinoma. 
Table 2 - Relationship between CXCR4 expression and clinico-morphological parameters

\begin{tabular}{|c|c|c|c|c|c|}
\hline Clinico-morphological parameters & $\begin{array}{c}\text { No. of cases } \\
(n)\end{array}$ & $\begin{array}{c}\text { Low IRS CXCR4 cases } \\
n(\%)\end{array}$ & $\begin{array}{c}\text { High IRS CXCR4 cases } \\
n(\%)\end{array}$ & $x^{2}$ value & $p$ value \\
\hline \multicolumn{6}{|l|}{ Tumor location } \\
\hline Stomach & 10 & $6(60)$ & $4(40)$ & \multirow{6}{*}{ Non-valid } & \\
\hline Small intestine & 12 & $8(66.7)$ & $4(33.3)$ & & \\
\hline Appendix & 6 & $6(100)$ & $0(0)$ & & \\
\hline Right colon & 11 & $5(45.5)$ & $6(54.5)$ & & \\
\hline Left colon (including rectum) & 13 & $6(46.2)$ & $7(53.8)$ & & \\
\hline Hepatic metastasis & 19 & $9(47.4)$ & $10(52.6)$ & & \\
\hline \multicolumn{6}{|l|}{ Tumor grade } \\
\hline $\mathrm{G} 1$ & 28 & $21(75)$ & $7(25)$ & \multirow{3}{*}{7666.2} & \multirow{3}{*}{0.0216} \\
\hline G2 & 21 & $11(52.4)$ & $10(47.6)$ & & \\
\hline G3 & 22 & $8(36.4)$ & $14(63.6)$ & & \\
\hline \multicolumn{6}{|l|}{ Tumor type } \\
\hline $\begin{array}{r}\mathrm{G} 1 \mathrm{NET} \\
\end{array}$ & 27 & $20(74.1)$ & $7(25.9)$ & \multirow{6}{*}{8057.5} & \multirow{6}{*}{0.1531} \\
\hline G2 NET & 18 & $9(50)$ & $9(50)$ & & \\
\hline G3 NET & 7 & $3(42.9)$ & $4(57.1)$ & & \\
\hline NEC & 12 & $4(33.3)$ & $8(66.7)$ & & \\
\hline MiNEN & 3 & $1(33.3)$ & $2(66.7)$ & & \\
\hline AGCC & 4 & $3(75)$ & $1(25)$ & & \\
\hline \multicolumn{6}{|l|}{ Tumor stage } \\
\hline $\mathrm{I}-\mathrm{II}$ & 17 & $14(82.4)$ & $3(17.6)$ & \multirow{2}{*}{6012.1} & \multirow{2}{*}{0.0142} \\
\hline III-IV & 44 & $21(47.7)$ & $23(52.3)$ & & \\
\hline \multicolumn{6}{|l|}{ Regional lymph nodes } \\
\hline $\mathrm{pN} 0$ & 19 & $8(42.1)$ & $11(57.9)$ & \multirow{2}{*}{0.0382} & \multirow{2}{*}{0.8450} \\
\hline pN1 & 23 & $9(39.1)$ & $14(60.9)$ & & \\
\hline \multicolumn{6}{|l|}{ Lympho-vascular invasion } \\
\hline Absent & 17 & $14(82.4)$ & $3(17.6)$ & \multirow{2}{*}{6178.1} & \multirow{2}{*}{0.0129} \\
\hline Present & 25 & $11(44)$ & $14(56)$ & & \\
\hline \multicolumn{6}{|l|}{ Perineural invasion } \\
\hline Absent & 28 & $19(67.9)$ & $9(32.1)$ & \multirow{2}{*}{2421.1} & \multirow{2}{*}{0.1197} \\
\hline Present & 14 & $6(42.9)$ & $8(57.1)$ & & \\
\hline
\end{tabular}

AGCC: Adenocarcinoma ex-goblet cell carcinoid; $X^{2}$ : Chi-squared; CXCR4: C-X-C motif chemokine receptor 4; G: Grade; IRS: Immunoreactivity score; MiNEN: Mixed neuroendocrine non-neuroendocrine neoplasm; NEC: Neuroendocrine carcinoma; NET: Neuroendocrine tumor.

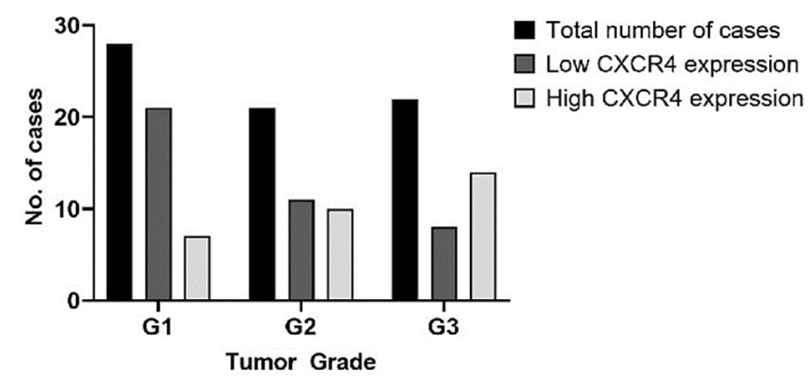

Figure 2 - Significant correlation was noted between CXCR4 expression and tumor grade. CXCR4: $C-X-C$ motif chemokine receptor 4 .

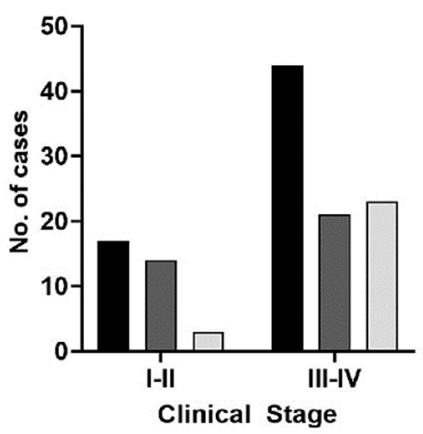

Total number of cases $\square$ Low CXCR4 expression $\square$ High CXCR4 expression

Figure 3 - Graphical representation of the relationship between CXCR4 expression and tumor stage. CXCR4: $C-X-C$ motif chemokine receptor 4.
Even though non-invasive techniques are very attractive and gain more and more attention in the field of oncology, immunohistochemistry remains the most affordable and practical method for determining different biological markers with impact in treatment and prognosis. A wide variety of tumors express the CXCR4 immunomarker, whose expression is usually associated with a negative prognosis and accelerated tumor progression.

To date, only a few studies have analyzed the CXCR4 expression in GI-NETs. In our study, we evaluated the IHC expression of the anti-CXCR4 monoclonal antibody, clone UMB2, from Abcam. Interpretation of immunoreactions for CXCR4 was difficult due to the heterogeneity of the staining patterns. In most cases, we noticed cytoplasmic or membranous pattern, but in rare cases we noticed the obvious staining of the nuclei. In our study, we considered positive immunoreactions, all cases with cytoplasmic, membrane or cytoplasmic and membrane immunostaining. High CXCR4 immunoexpression was defined by IRS between 4 and 12 . Out of 71 GI-NENs, 31 (43.7\%) cases had high CXCR4 immunoexpression. High CXCR4 tumors were more frequent in women $(54.8 \%)$, with the average age of the patients being 61.2 years, higher than the average age of patients with low CXCR4 immunoexpression, which was 58.9 years.

Kaemmerer et al. established an inversely proportional 
relationship between CXCR4 expression and tumoral grade, hypothesis supported by our results [7]. Welldifferentiated G1 NETs had low CXCR4 expression in $75 \%$ of the cases, while G3 NETs and NECs had high CXCR4 expression in $63.6 \%$ of the cases.

Additionally, some authors have identified a significant correlation between CXCR4 expression and the Ki-67 PI, a statement also supported by our study. Seven cases of G1 NET and eight cases of G2 NET with high CXCR4 expression were identified, despite the low $\mathrm{Ki}-67$ rate. Our results also correlate CXCR4 expression with tumor type, as $74.1 \%$ of the G1 NETs had low IRS and $66.7 \%$ of NECs had high IRS. Therefore, we can affirm that IRS proportionately increases with tumor aggressivity, defining CXCR4 as a negative prognostic factor in GI-NENs. However, in G3 NET there is no clear distinction between cases with high IRS and low IRS, which may mean that for this group of lesions, CXCR4 probably has no therapeutic applicability.

Regarding the origin of NETs, the study of Mai et al. reports that tumors with intense CXCR4 expression develop most commonly in the appendix and colon [8]. However, from our results we observe that tumors with low IRS for CXCR4 were located more frequently in the small intestine (66.7\%), stomach (60\%) and especially appendix (100\%). In all appendix tumors, the calculated IRS was 0 . Liver metastases showed higher CXCR4 immunoreactions expression $(52.5 \%)$ more frequently than primary tumors $(40.4 \%)$.

In contradiction with the study conducted by Deschamps et al., which states that CXCR4 expression correlates positively with perineural invasion and lymph node metastases [20], the results of our study do not confirm this conclusion. Our results show the association between low IRS, early clinical stages $(82.4 \%)$ and the absence of lympho-vascular invasion (82.4\%). CXCR4 expression does not correlate with $\mathrm{pN}$ stage and perineural invasion.

CXCR4 expression was identified in the intratumoral microvessels. Consequently, a hypothesis that CXCR4 intervenes in tumor neoangiogenesis and that it represents a possible target in antiangiogenic therapy was formulated [21].

Although numerous treatment regimens have been studied, therapeutic options for patients with NENs remain limited. This is the particular case of patients with G3 NET and NEC, which requires the introduction of new targeted therapies, more effective than those currently existing. Peptide receptor radionuclide therapy (PRRT) is an attractive therapeutic option for patients with inoperable or disseminated NENs. This therapy uses cytotoxic radiation against tumoral cells and is usually applied to patients with tumors that express SSTRs. Recently, an attempt has been made to apply this technique against the CXCR4 ligand using ${ }^{68} \mathrm{Ga}$-Pentixafor $/{ }^{177} \mathrm{Lu} /{ }^{90} \mathrm{Y}$-Pentixather or scandium and terbium radioisotopes [22].

The CXCR4/CXCL12/CXCR7 activation pathway is overexpressed at the tumor level. CXCL12 activates CXCR4, which subsequently activates the mTOR signaling pathway, thus generating an additional effect on tumor cell proliferation. To counteract this effect, two therapeutic agents AMD3100 (Plerixafor) and RAD001 (Everolimus) were developed, with inhibitory effect on tumor growth [23]. Given the important role that CXCR4 plays in tumor development and dissemination, more studies are needed on this topic to develop targeted, effective therapies that can prolong the survival of patients with advanced NENs.

\section{a Conclusions}

Our study confirms that CXCR4 is usually expressed in GI-NENs. These results prove that CXCR4 has a crucial role in NEN tumorigenesis. An important exception were the NENs of the appendix, where CXCR4 was always negative. In our retrospective study, the high CXCR4 expression was correlated with NENs in advanced stages as well as with high grade tumors. Consecutively, we consider that a high IRS represents an important negative prognostic factor for GI-NENs. CXCR4 high expression was also associated with lympho-vascular invasion. Therefore, we recognize the necessity of additional studies which could demonstrate if anti-CXCR4 therapy has an anti-angiogenetic effect for patients with lympho-vascular invasion. Our study also identified patients with G1 and G2 NETs with high IRS values. This could help us select patients with G1 and G2 NETs, that are prone to have an unfavorable evolution, and that could be more actively surveilled. For these patients, treatment with CXCR4 inhibitors may serve as a significant therapeutic option which is to be validated in prospective clinical studies.

\section{Conflict of interests}

The authors declare that they have no conflict of interests.

\section{References}

[1] Ahmed M. Gastrointestinal neuroendocrine tumors in 2020. World J Gastrointest Oncol, 2020, 12(8):791-807. https:// doi.org/10.4251/wjgo.v12.i8.791 PMID: 32879660 PMCID: PMC7443843

[2] Hashmi AA, Ali J, Khan K, Ahmed O, Rehman AU, Irfan M, Haroon S, Asif MG. Clinicopathological spectrum of primary and metastatic neuroendocrine neoplasms. Cureus, 2020, 12(11):e11764. https://doi.org/10.7759/cureus.11764 PMID: 33409012 PMCID: PMC7779123

[3] Wang R, Zheng-Pywell R, Chen HA, Bibb JA, Chen H, Rose JB. Management of gastrointestinal neuroendocrine tumors. Clin Med Insights Endocrinol Diabetes, 2019, 12:1179551419 884058. https://doi.org/10.1177/1179551419884058 PMID: 31695546 PMCID: PMC6820165

[4] Cockburn A, Rege TA. Gastrointestinal neuroendocrine lesions. In: Noffsinger AE (ed). Fenoglio-Preiser's gastrointestinal pathology. $4^{\text {th }}$ edition, Wolters Kluwer, Philadelphia, USA, 2017, 1004-1068. https://pathology.Iwwhealthlibrary.com/book.asp $x$ ?bookid $=2648$

[5] Nagtegaal ID, Odze RD, Klimstra D, Paradis V, Rugge M, Schirmacher P, Washington KM, Carneiro F, Cree IA; WHO Classification of Tumours Editorial Board. The 2019 WHO Classification of tumours of the digestive system. Histopathology, 2020, 76(2):182-188. https://doi.org/10.1111/his.13975 PMID: 31433515 PMCID: PMC7003895

[6] Mafficini A, Scarpa A. Genetics and epigenetics of gastroenteropancreatic neuroendocrine neoplasms. Endocr Rev, 2019, 40(2):506-536. https://doi.org/10.1210/er.2018-00160 PMID: 30657883 PMCID: PMC6534496

[7] Kaemmerer D, Träger T, Hoffmeister M, Sipos B, Hommann M, Sänger J, Schulz S, Lupp A. Inverse expression of somatostatin and CXCR4 chemokine receptors in gastroenteropancreatic neuroendocrine neoplasms of different malignancy. Oncotarget, 2015, 6(29):27566-27579. https://doi.org/10.18632/oncotarget. 4491 PMID: 26259237 PMCID: PMC4695009

[8] Mai R, Kaemmerer D, Träger T, Neubauer E, Sänger J, Baum RP, Schulz S, Lupp A. Different somatostatin and CXCR4 chemokine receptor expression in gastroenteropancreatic neuroendocrine neoplasms depending on their origin. Sci Rep, 
2019, 9(1):4339. https://doi.org/10.1038/s41598-019-39607-2 PMID: 30867449 PMCID: PMC6416272

[9] Misdraji J, Carr N, Pai R. Tumours of the appendix. Goblet cell adenocarcinoma. In: WHO Classification of Tumours Editorial Board (ed). WHO Classification of Tumours. Digestive System Tumours. $5^{\text {th }}$ edition, vol. 1, World Health Organization (WHO), International Agency for Research on Cancer (IARC) Press, Lyon, France, 2019, 149-151. https://publications.iarc.fr/579

[10] Murnyák B, Hortobágyi T. Immunohistochemical correlates of TP53 somatic mutations in cancer. Oncotarget, 2016, 7(40): 64910-64920. https://doi.org/10.18632/oncotarget.11912 PMID: 27626311 PMCID: PMC5323125

[11] Nielsen K, Binderup T, Langer SW, Kjaer A, Knigge P, Grøndahl V, Melchior L, Federspiel B, Knigge U. P53, Somatostatin receptor $2 a$ and Chromogranin A immunostaining as prognostic markers in high grade gastroenteropancreatic neuroendocrine neoplasms. BMC Cancer, 2020, 20(1):27. https://doi.org/10.1186/s12885-019-6498-z PMID: 31924180 PMCID: PMC6953213

[12] Remmele W, Stegner HE. [Recommendation for uniform definition of an immunoreactive score (IRS) for immunohistochemica estrogen receptor detection (ER-ICA) in breast cancer tissue]. Pathologe, 1987, 8(3):138-140. PMID: 3303008

[13] Smith H, Whittall C, Weksler B, Middleton J. Chemokines stimulate bidirectional migration of human mesenchymal stem cells across bone marrow endothelial cells. Stem Cells Dev, 2012, 21(3):476-486. https://doi.org/10.1089/scd.2011.0025 PMID: 21513440

[14] Howard OMZ, Ben-Baruch A, Oppenheim JJ. Chemokines: progress toward identifying molecular targets for therapeutic agents. Trends Biotechnol, 1996, 14(2):46-51. https://doi.org/ 10.1016/0167-7799(96)80920-6 PMID: 8746116

[15] Chatterjee S, Azad BB, Nimmagadda S, Morgan RH. The intricate role of CXCR4 in cancer. Adv Cancer Res, 2014, 124:31-82. https://doi.org/10.1016/B978-0-12-411638-2.000 02-1 PMID: 25287686 PMCID: PMC4322894

[16] Balkwill F. Cancer and the chemokine network. Nat Rev Cancer, 2004, 4(7):540-550. https://doi.org/10.1038/nrc1388 PMID: 15229479

[17] Wester HJ, Keller U, Schottelius M, Beer A, Philipp-Abbrederis K, Hoffmann F, Šimeček J, Gerngross C, Lassmann M, Herrmann K,
Pellegata N, Rudelius M, Kessler H, Schwaiger M. Disclosing the CXCR4 expression in lymphoproliferative diseases by targeted molecular imaging. Theranostics, 2015, 5(6):618630. https://doi.org/10.7150/thno.11251 PMID: 25825601 PMCID: PMC4377730

[18] Morse DL, Gillies RJ. Molecular imaging and targeted therapies. Biochem Pharmacol, 2010, 80(5):731-738. https://doi.org/ 10.1016/j.bcp.2010.04.011 PMID: 20399197 PMCID: PMC 2925390

[19] Weich A, Werner RA, Buck AK, Hartrampf PE, Serfling SE, Scheurlen M, Wester HJ, Meining A, Kircher S, Higuchi T, Pomper MG, Rowe SP, Lapa C, Kircher M. CXCR4-directed $\mathrm{PET} / \mathrm{CT}$ in patients with newly diagnosed neuroendocrine carcinomas. Diagnostics (Basel), 2021, 11(4):605. https://doi.org/ 10.3390/diagnostics11040605 PMID: 33805264 PMCID: PMC8067200

[20] Deschamps L, Bacha D, Rebours V, Mebarki M, Bretagnol F, Panis Y, Bedossa P, Ruszniewski P, Couvelard A. The expression of the hypoxia markers CA9 and CXCR4 is correlated with survival in patients with neuroendocrine tumours of the ileum. Neuroendocrinology, 2012, 95(3):214-222. https://doi.org/10. 1159/000329873 PMID: 22133596

[21] Kajtazi Y, Kaemmerer D, Sänger J, Schulz S, Lupp A. Somatostatin and chemokine CXCR4 receptor expression in pancreatic adenocarcinoma relative to pancreatic neuroendocrine tumours. J Cancer Res Clin Oncol, 2019, 145(10): 2481-2493. https://doi.org/10.1007/s00432-019-03011-0 PMID: 31451931

[22] Nicolas GP, Morgenstern A, Schottelius M, Fani M. New developments in peptide receptor radionuclide therapy. J Nucl Med, 2019, 60(2):167-171. https://doi.org/10.2967/jnumed. 118.213496 PMID: 30573642

[23] Circelli L, Sciammarella C, Guadagno E, Tafuto S, del Basso de Caro M, Botti G, Pezzullo L, Aria M, Ramundo V, Tatangelo F, Losito NS, leranò C, D'Alterio C, Izzo F, Ciliberto G, Colao A, Faggiano A, Scala S. CXCR4/CXCL12/CXCR7 axis is functional in neuroendocrine tumors and signals on mTOR. Oncotarget, 2016, 7(14):18865-18875. https://doi.org/10.18632/oncotarget. 7738 PMID: 26934559 PMCID: PMC4951335

\section{Corresponding author}

Sorina Maria Tăban, Associate Professor, MD, PhD, Anapatmol Research Center, Victor Babeş University of Medicine and Pharmacy, 2 Eftimie Murgu Square, 300041 Timişoara, Romania; Phone +40755-121 602, e-mail: sorinataban@yahoo.com

Received: July 5, 2021

Accepted: July 16, 2021 